\title{
Performance Evaluation of IEEE 802.15.4 Sensor Networks in Industrial Applications
}

\author{
Wan Du*, David Navarro and Fabien Mieyeville \\ Lyon Institute of Nanotechnology (INL), University of Lyon UMR5270 - CNRS, Ecole Centrale de Lyon, Ecully, \\ F-69134, France
}

\begin{abstract}
SUMMARY
Wireless sensor networks (WSNs) have been widely applied in industrial applications especially since the release of IEEE 802.15.4 standard. By participating in an automobile project in which an IEEE 802.15.4 based sensor and actuator network is deployed to measure and control the vibrations of an automotive system, we need to study many metrics of IEEE 802.15.4 sensor networks (e.g., packet delivery rate,latency and energy consumption) under various sampling rates. In order to provide detailed modeling of hardware and software as well as network behaviors on each sensor node, we conduct plenty of experiments on a SystemC-based WSN simulator IDEA1 which supports the hardware and software co-simulation of sensor nodes with certain flexibility of abstraction level. Compared with the existing works on performance evaluation of IEEE 802.15.4 protocols, the main contributions of this paper are the comprehensive studies of both beacon-enabled and nonbeacon-enabled modes under various parameter settings and the beacon tracking synchronization mechanism in the IEEE 802.15.4 standard which is ignored in most previous works. Additionally, the in-depth analysis of simulation results enables us to find the best parameter configurations to different traffic loads and application requirements which can be used as general experiences for other applications. Copyright (C) 0000 John Wiley \& Sons, Ltd.
\end{abstract}

Received ...

KEY WORDS: Wireless sensor network; IEEE 802.15.4; Evaluation; Industrial Application

\section{INTRODUCTION}

Our group has participated in an industrial project, Mechanics@Lyon [1], which is intended to improve the internal comfort (reduce the vibration and noise) by identifying and integrating the current intelligent control technologies in automotive systems. A wireless sensor and actuator network is deployed on an automobile to measure and control the vibration. The sensor network is composed of several nodes and a coordinator. The nodes periodically measure the accelerations of their given positions by a piezoelectric sensor and transmit their sensor data to the coordinator,

\footnotetext{
${ }^{*}$ Correspondence to: Wan Du, wan3102@gmail.com. This work is done during his PhD studies at University of Lyon, France. Now he is working as a research fellow at Nanyang Technological University, Singapore.

Copyright (c) 0000 John Wiley \& Sons, Ltd.

Prepared using dacauth.cls [Version: 2010/03/27 v2.00]
} 
which is a more powerful node in charge of collecting the sensor data from all nodes. Based on the analysis of collected data, some control actions are executed by the actuator network.

In this industrial application, some preliminary designs based on the existing node hardware platforms and network protocols shall be evaluated at first. We need to study many metrics of this sensor network (e.g., packet delivery rate and latency) under various sampling rates and consider the power consumption of sensor nodes since they are wireless communication modules with small size to minimize the impact of measurement hardware on original automobile system. Wireless sensor networks (WSNs) have been widely used in industrial automations [2] since the IEEE 802.15.4 standard has been released [3]. In this paper, we evaluate the performance of IEEE 802.15.4 protocols based on MICAz motes [4] through simulation since it is the most direct and efficient method to perform this study compared with mathematical analysis or experimental testbeds.

Many general-purpose network simulators like NS-2 [5] and OMNeT++ [6] have been used in WSN simulations. For example, a Petri-net based simulation model of a wireless mobile ad hoc network is developed in [7]. The simulation model was implemented in terms of a class of extended Petri nets to explicitly represent parallelism of events and processes in the WLAN as a distributed system. Generally, the network simulators have the advantages of extensibility, heterogeneity support and easy-to-use. However, the energy consumption estimation is usually based on some simple assumptions, for example, the processor and Radio-frequency (RF) transceiver have the same operating states. On the other hand, several Operating System (OS) emulators (e.g., TOSSIM [8]) and Instruction Set Simulators (ISS) (e.g., Avrora [9]) are developed to emulate the embedded software execution on sensor nodes. They can provide a high timing accuracy of software execution and thus energy consumption of protocols. However, they suffer from the scalability limitation and are generally constrained to specific pre-defined hardware platforms or operating systems.

In order to support the hardware and software (HW/SW) co-simulation of sensor nodes with certain flexibility of abstraction level, we use a SystemC-based WSN simulator named IDEA1 (hIerarchical DEsign plAtform for sensOr Networks Exploration) [10] in this study. It is a sensor node simulator which allows rapid performance evaluation of WSN applications and protocols at system-level. SystemC [11] is a System-Level Description Language (SLDL) which provides native supports to model concurrency, structural hierarchy, interrupts and synchronization of embedded systems. To the best of our knowledge, IDEA1 is the first SystemC-based WSN simulator validated with experimental measurements and evaluated comprehensively with other simulators [10].

In this work, the sensor node hardware platform, MICAz, is modeled by SystemC in the IDEA1 simulation environment. Both the slotted CSMA-CA protocol in beacon-enabled mode and the unslotted CSMA-CA protocol in nonbeacon-enabled mode are implemented and studied in the aspects of packet delivery rate, latency, power consumption per node and energy consumption per packet. Two synchronization mechanisms in the IEEE 802.15.4 standard, the beacon tracking and non beacon tracking, are evaluated. The effect of various parameter settings, especially the beacon order and the superframe order, are analyzed under different traffic loads. Compared to the pervious works on performance evaluation of IEEE 802.15.4 [12-22], this paper has many unique features, as summarized as follows.

- The evaluation is based on a novel simulation tool which supports hardware/software cosimulation. Accurate energy consumption prediction based on exact models of hardware 
components of sensor nodes is realized. It considers the current consumption of not only each operation state of sensor nodes but also the transitions between different states.

- Besides the slotted CSMA-CA protocol in beacon-enabled mode and the unslotted CSMACA protocol in nonbeacon-enabled mode, we also study the beacon tracking and non beacon tracking synchronization mechanisms in the IEEE 802.15.4 standard which is ignored in most previous works.

- This study is driven by a real industrial application about the measurement and control of automobile vibrations. However, the common features of industrial application like star topology and various traffic load are summarized and the simulation results are comprehensively analyzed. The conclusions can thus be used in general applications with the same star topology.

The rest of this paper is organized as follows. Section 2 summarizes the related works. We briefly review IEEE 802.15.4 in Section 3. The implementation of IEEE 802.15.4 in IDEA1 and the simulation configuration are presented in Section 4. The performances of IEEE 802.15.4 sensor networks are evaluated under various traffic loads and parameter settings in Section 5. We conclude this paper in Section 6.

\section{RELATED WORKS}

Since the first release of the IEEE 802.15.4 standard in 2003 and revised in 2006, many performance evaluation works of this protocol have been published, including analytical studies [23] [24] and simulations. Since the analytical studies normally are based on some assumptions and thus cannot fully take into account all possible situations in reality, in this work, we focus on the performance evaluation of IEEEE 802.15.4 sensor networks using simulations.

The first performance evaluation of IEEE 802.15.4 is presented in [12] and [13] based on an NS2 implementation model, while an energy model is later added in [14]. The performance of IEEE 802.15.4 has been compared with IEEE 802.15.4 in the aspects of packet delivery and latency. The other performance of IEEE 802.15.4, like association efficiency, orphaning, collision avoidance and GTS data transmissions have also been studied. These studies have been extended recently by many works considering the effect of parameter settings and special applications.

The performance of IEEE 802.15.4 slotted CSMA-CA algorithm, including packet delivery ratio, latency and energy consumption, is studied by NS-2 simulation in [15]. It also considers the difference between tracking and non-tracking synchronization mechanisms. However, it is an early work which based on a simple energy consumption model and did not take into account the effect of different parameter settings. Petrova et al. [16] analyze the properties and performance of IEEE 802.15.4 through measurement of the RSSI, PER. They also evaluate the performance of IEEE 802.15.4 slotted CSMA-CA through NS-2 simulation based on the model in [12]. However, only limited settings and metrics are considered.

Rohm et al. [17] analyze the effect of different configuration of IEEE 802.15.4 MAC protocol parameters on the performance of non-beacon mode operations under different traffic loads and levels of interference from hidden terminals, especially with the metrics of packet loss probability and latency. Chen et al. [18] evaluates the performance of IEEE 802.15.4 in industrial sensor 
network applications, especially in the aspects of end-to-end latency and energy consumption. They develop an implementation of IEEE 802.15.4 in OMNeT++ to investigate the effect of parameters like beacon order and superframe order to different traffic loads on sensor networks of star topology. We further improve this work by an more detailed energy model benefiting from SystemC-based simulation with considerations of both hardware components and software executions. Some preliminary results, especially the model development and energy consumption modeling, have been presented in [25] [26]. In this paper, we also study the performance of beacon tracking mechanisms and non-beacon mode with unslotted CSMA-CA algorithm.

Xia et al. [19] study IEEE 802.15.4 for cyber physical systems over wireless sensor and actuator networks, especially considering the requirements on quality of service (QoS) characterized by several metrics like data rate, packet loss rate and end-to-end delay. They also focus on a star network with eight sensor nodes collecting data and transmitting them to a central sink node. However, the energy consumption is not taken into account in this work which is one of the most important factors in the design of IEEE 802.15.4 sensor networks. The theoretical throughput and delay bounds of the unslotted version of chirp spread spectrum PHY-based 802.15.4a is analyzed in [27]. The performance of 802.15.4a and 802.15.4 is compared in terms of throughput and delay. Nordin et al. [20] conduct simulations to study the beacon collision problems in the co-located IEEE 802.15.4-based Networks where more than one wireless personal area networks are deployed. A medium access control protocol is proposed in [28] based on framed slotted aloha for these networks. Many metrics, such as probable packet sizes, energy consumptions, battery lifetime and the success rate are studied by simulations.

Besides the industrial applications, IEEE 802.15.4 has also been used in many other fields, such as Wireless Body Area Network (WBAN) [21] and electrocardiogram monitoring [22]. The features of low power consumption and data rate have made IEEE 802.15.4 being an active candidate for these applications. In [29], the performance of IEEE 802.15.4 in u-healthcare applications is analyzed through experiments. Additionally, simulation has been widely used in the performance evaluation of other wireless protocols. For example, a model for WiMAX platform on OPNET has been used to evaluate the adaptation at the physical layer of the transmission in WiMAX OFDMA structure [30], and an IEEE 802.11 model on NS-2 has been developed in [31] with assumptive physical parameters to evaluate the throughput performance of a novel MAC protocol. The system performance of wireless sensor and actuator network to detect and extinguish a fire in a burning wildland is analyzed through simulation in [32].

Compared with the above works, the main contributions of this paper are the comprehensive studies of both beacon-enabled and nonbeacon-enabled modes as well as the beacon tracking mechanism in IEEE 802.15.4 sensor networks in many aspects of packet transmissions and the evaluation of energy consumption by using detail power consumption profiling and SystemC-based hardware/software co-simulation. Additionally, we provide in-depth analysis of simulation results to find the best parameter configurations to different traffic loads in industrial applications. 


\section{BRIEF OVERVIEW OF IEEE 802.15.4}

The IEEE 802.15.4 standard [3] specifies the PHY and MAC layers that are the basis for many upper layer protocol standards (e.g., ZigBee [33]). It has been widely used in WSN application since it is designed for low data rate, short distance, and low power consumption applications in conformity with WSN constraints.

The MAC layer controls access to the radio channel using CSMA-CA mechanism. IEEE 802.15.4 MAC layer supports two operational modes: the nonbeacon-enabled mode with unslotted CSMA$\mathrm{CA}$ and the beacon-enabled mode with beacons periodically sent by the coordinator to synchronize nodes and to delimit a superframe during which all transmissions must occur.

For the unslotted CSMA-CA algorithm, firstly, the number of backoff (NB) is initialized to 0 . Then the algorithm starts counting down a random number of backoff periods. A backoff period, called aUnitBackoffPeriod, is equal to 20 symbols. One symbol is the transmission time of 4 bits, which is $16 \mu \mathrm{s}$ for a data rate of $250 \mathrm{kbps}$. When the timer expires, the algorithm performs channel assessment. If the channel is idle, it starts transmitting; otherwise, NB is incremented. If NB does not reach the maximum number of backoff (macMaxCSMABackoff), the algorithm goes back to delay a random number of backoff periods again; otherwise, the channel access operation fails.

In slotted CSMA-CA algorithm, the operations of different sensor nodes are synchronized. The backoff period boundaries of every sensor node shall be aligned with a superframe slot boundaries of the coordinator. Beacon packets are transmitted periodically by the coordinator to describe the superframe structure, as shown in Fig. 1.

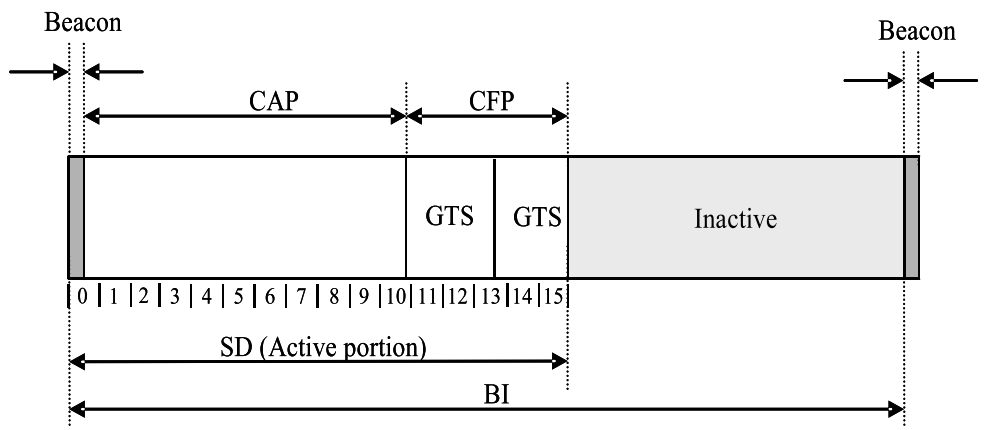

Figure 1. The typical structure of a superframe [3]

Beacon Interval (BI) defines the superframe length, which includes an active period and an optional inactive period. Superframe Duration (SD) presents the length of active period. They are determined by two parameters, Beacon Order (BO) and Superframe Order (SO).

$$
\begin{gathered}
B I=\text { aBaseSuperframe } \cdot 2^{B O}, 0 \leq B O \leq 14 \\
S D=\text { aBaseSuperframe } \cdot 2^{S O}, 0 \leq S O \leq B O \leq 14
\end{gathered}
$$

The minimum duration of a superframe (aBaseSuperframeDuration) is fixed to 960 symbols corresponding to $15.36 \mathrm{~ms}$, assuming $250 \mathrm{kbps}$ in the $2.4 \mathrm{GHz}$ frequency band. The active portion consists of two periods, namely contention access period (CAP) and contention free period (CFP). 
During CAP, nodes use the slotted CSMA-CA algorithm to access the channel. During CFP, many GTSs (up to 7) can be allocated, which allow the node to operate on the channel that is dedicated exclusively to it. In [34], an GTS allocation mechanism in IEEE 802.15. 4 is proposed to achieve an reasonable trade-off between energy and delay.

In the IEEE 802.15.4 standard, there are two synchronization mechanisms in the beacon-enabled mode: beacon tracking and non beacon tracking (presented as noTracking mode). If tracking is specified, the node shall attempt to acquire the beacon and keep tracking it by regular and timely activation of its receiver. After the transmission, if it has no sensor data to send, it will go to sleep mode, but will keep tracking the beacon packet. If tracking is not specified, the node shall attempt to acquire the beacon only once it needs to send and terminate the tracking after the next beacon.

\section{SIMULATION IN IDEA1}

IDEA1 [10] [35] is developed in SystemC and C++. The sensor node is modeled in SystemC and the interconnections among nodes are implemented in C++. IDEA1 includes a library that contains many implementations of existing hardware platforms, such as ATMega128 microcontroller, TI CC2420 transceiver and Microchip MRF24J40 transceiver. Both CC2420 and MRF24J40 implement IEEE 802.15.4 PHY and MAC protocol. The differences between these two chips are the MAC layer implementation. MRF24J40 implements almost all MAC specifications of the standard; however, some MAC functions need to be done by software. This feature impacts the energy consumption of these two chips slightly. In this work, we analyze MICAz motes with ATMega128 microcontroller and TI CC2420 transceiver, since this mote is more widely used WSN applications. Its main hardware component, CC2420, has also been used in many other hardware platforms, like TelosB.

\subsection{Architecture of IDEAI}

IDEA1 is a component-based simulator. Each hardware component is modeled as an individual module of SystemC. A typical model of sensor node is composed by four parts, i.e., sensor, microcontroller, transceiver and battery. A more detailed illustration can be found in [10]. For hardware modeling, sensor is simulated as a stimuli generator that is an interface specifying how the physical parameters in the environment vary in spatial and temporal terms. The processing unit converts the analog signal from the sensor module into digital format by a built-in Analog to Digital Converter (ADC), processes the data and sends the packet to the RF transceiver via a Serial Peripheral Interface (SPI) bus which is a standard communication type between microcontroller and transceiver. The RF transceiver emits the packets in the network by different media access protocols. Some connections are used by the RF transceiver to report interrupts (INT) to the processing unit, such as a receipt of packet, Clear Channel Assessment (CCA), etc. The processing unit can also wake up or reset the RF transceiver by two connections relatively. The processing unit and RF transceiver are modeled as Finite State Machines (FSMs). During simulation, the state transition traces of each component are recorded. Each state of the hardware components is associated with a Current Consumption (CC) based on experimental measurements and their data sheets. The duration and current consumption of each transition between two states are also identified. Based on this 
information, the battery module calculates the energy consumption of each component and its residual capacity according to particular battery models (e.g., linear model, discharge rate dependent model and relaxation model [36]).

All sensor nodes are interconnected with each other through a network model which relays messages from one node to others and models the radio signal propagation. Two propagation models have been implemented in IDEA1. Other propagation models can be easily added. For example, a new composite channel model is proposed for the performance analysis of shadowed fading channels in [37]. The SystemC kernel acts as the simulation engine. It schedules the execution of processes and updates the state of all modules at every simulation cycle. The simulation of SystemC can be divided in three distinct major phases, includes elaboration, simulation and postprocessing [38]. All the setting and control of these phases are implemented in a sc_main function, which is the start point of the SystemC simulation program. During the elaboration phase, all the modules are initialized; the relative ports are connected by signals. After elaboration, the simulation execution begins with a call of the sc_start function. The simulation kernel will update the values of all signals and invoke all the active processes at every simulation cycle. An active process goes into the suspended state after it completes its operation or reaches a wait statement. Once all the active processes are invoked, simulator time advances a simulation cycle. Because all the active processes are invoked at the same simulator time, it creates an illusion of concurrency. Finally, when the simulator time reaches the point that the application sets, the simulation stops and all the objects are deleted.

\subsection{IEEE 802.15.4 Implementation}

For MICAz motes, the MAC layer specifications are implemented as software operations of microcontroller since the transceiver of MICAz mote (TI CC2420) does not provide hardware support of the IEEE 802.15.4 MAC layer. The native support of hardware and software co-modeling embedded systems by SystemC, such as primitives to model the concurrency, interrupts, structural hierarchy and synchronization, enables us to easily model the HW/SW of sensor nodes within one environment. In the implementation of IDEA1, if an interrupt occurs when the microcontroller is at SENSING state, it will be ignored; however, when the microcontroller is at other states, the microcontroller will stop what it is doing and handle the sensing interrupt immediately. It will resume its task when the sensing operation finishes.

When the microcontroller is in TX state, the node (both microcontroller and RF transceiver) needs to perform specific communication protocols in order to access channel and avoid collisions. The protocols can be implemented either by RF transceiver with hardware or by microcontroller with software. For MICAz, the MAC protocol has to be implemented by microcontroller and thus the TX state of microcontroller is divided into many sub-states. When entering to TX state, the microcontroller first backoffs a random duration and checks the channel. If the channel is free, it will start transmitting. The backoff period boundaries should be aligned with the superframe slot boundaries and the microcontroller should ensure that the transceiver commences all of its transmissions on the boundary of a backoff period. One backoff period includes 20 symbols, corresponding to $320 \mu \mathrm{s}$. For the unslotted CSMA-CA algorithm, nodes do not have to locate the backoff boundary and the clear channel assessment needs to be performed only once. 
Table I. Current consumptions of MICAz mote [39] [40]

\begin{tabular}{|ll|l|l|}
\hline Microcontroller (ATMEL ATMega128) & \multicolumn{2}{|c|}{ Transceiver (TI CC2420) } \\
\hline PowerDown & $0.3 \mu \mathrm{A}$ & PowerDown & $17 \mu \mathrm{A}$ \\
PowerSaving & $8.9 \mu \mathrm{A}$ & IDLE & $426 \mu \mathrm{A}$ \\
IDLE & $4 \mathrm{~mA}$ & RX & $18.8 \mathrm{~mA}$ \\
Active & $9 \mathrm{~mA}$ & TX(0dBm) & $17.4 \mathrm{~mA}$ \\
& & TX(-5dBm) & $13.9 \mathrm{~mA}$ \\
& TX(-10dBm) & $11.2 \mathrm{~mA}$ \\
& TX(-25dBm) & $8.5 \mathrm{~mA}$ \\
& PowerDown -> IDLE & $426 \mu \mathrm{A}(1 \mathrm{~ms})$ \\
& IDLE - $>$ RX & $18.8 \mathrm{~mA}(192 \mu \mathrm{s})$ \\
& IDLE - $>$ TX & $6.7 \mathrm{~mA}(192 \mu \mathrm{s})$ \\
\hline
\end{tabular}

\subsection{Energy Consumption Estimation}

In IDEA1, each state of the main hardware components of sensor nodes is associated with a current load. The duration and current consumption of each transition between two states are also identified. During the simulation, the states of these components are updated according to software execution and network events. Based on this information, the battery module calculates the energy consumptions of each component as well as the network lifetime during runtime. In this work, a linear battery model is used. The current consumptions of the main operation modes of the hardware components of MICAz are summarized according to the data sheets of their main hardware components [39] [40], as presented in Table I.

IDEA1 also provides a graphical user interface to facilitate users to configure system, control simulation and analyze results. It is useful for debugging the energy consumption. There are three kinds of simulation output in IDEA1, including simulation log, event sequence tracing file and statistic results calculated during simulation. The simulation log is used to debug the model implementations and show the network behaviors. During the simulation is running, the states of every hardware components and the variables are updated continually. Depending on the event sequence tracing, we can also verify the timing accuracy at cycle accurate level.

\section{SIMULATION RESULTS}

In this section, we evaluate the performance of using IEEE 802.15.4 in our industrial application. As introduced in Section 1, a sensor network of eight nodes and one coordinator are deployed on a vehicle. Since every node can reach the coordinator through single hop radio transmission, the network forms a star topology. Nodes send sensor data (one byte) to the coordinator periodically by using different protocols in IEEE 802.15.4. The TX power of transceiver is set to $0 \mathrm{dBm}$. Nodes go to SLEEP mode after the transmission finishes. They are woken up by a built-in timer. It is clocked by an external oscillator so as to continue to run during the sleep mode of microcontroller and generate an interrupt on overflow.

Many cases with various configurations of parameters (mainly $B O$ and $S O$ ) and different sample rates have been studied. Other parameters of the CSMA-CA algorithms (e.g., macMinBE, macMaxCSMABackoffs, macMaxFrameRetries, etc.) are set to the values defined by default in the IEEE 802.15.4 standard. Each simulation includes 10000 samples, for example, an application last 10000 s if the sample rate is 1 . Each case is simulated 100 times with random seeds for the random 
function of the backoff slot number. Four metrics are used to evaluate the network performance. They are defined as follows.

- Packet Delivery Rate (PDR): PDR is the ratio of the number of packets successfully received by coordinator to the number of generated packets.

- Average Latency $(A L)$ : Latency of a sensor data is the duration from the sensor data read by a node to the packet received by the coordinator. $A L$ is an average latency of all packets that successfully received by the coordinator.

- Average Power Consumption (APC): $A P C$ is utilized to measure the average power consumption per node which is a basic parameter to predict the lifetime of a sensor node and the network.

- Energy Consumption per Packet (ECPkt): ECPkt is the average energy consumed for successfully transmitting one packet.

\subsection{Slotted CSMA-CA with Fixed SO and Various BO}

To investigate the impact of different combination $B O$ and $S O, B O$ is set to 0,1 and 2 respectively and $S O$ is set to 0 for all simulations.

5.1.1. Packet Delivery Rate The simulation results of packet delivery rate are presented in Fig. 2, where Tracking presents the simulations of beacon tracking mechanism and noTracking refers to the simulations without beacon tracking mechanism. Firstly, we explain the change tendency of notracking mode to traffic loads. Finally, we study the difference between the notracking and tracking modes.

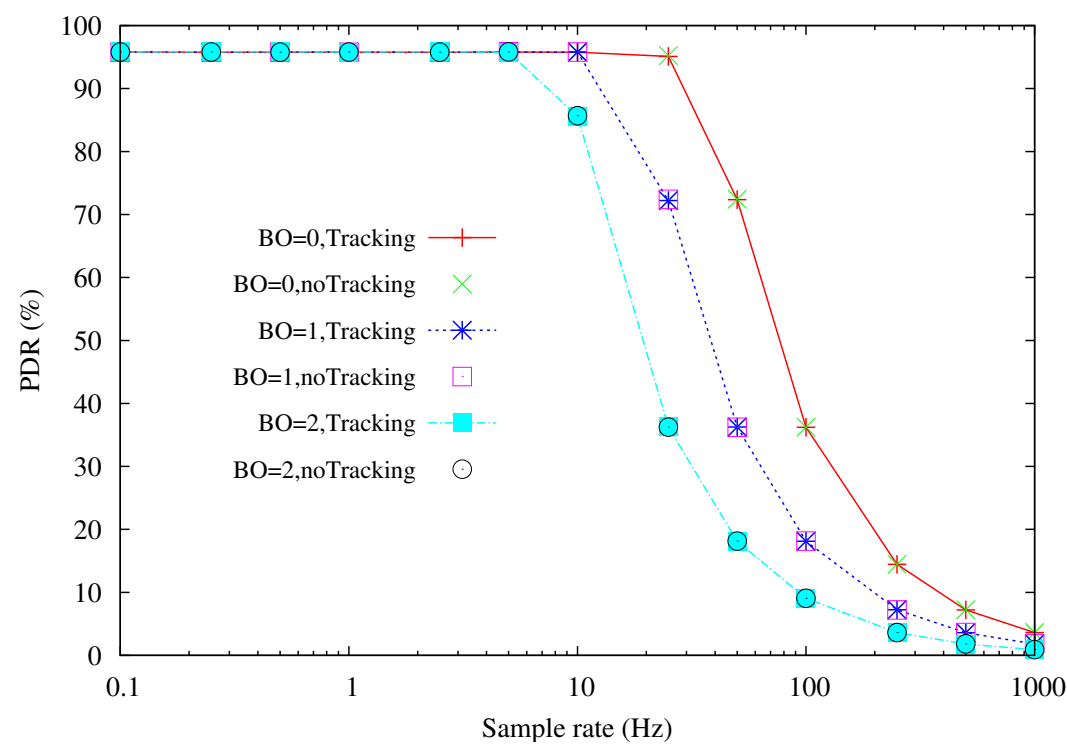

Figure 2. Packet delivery rate of slotted CSMA-CA with fixed $S O$ and various $B O$

Effect of traffic load. In the light traffic load area on the left side of Fig. 2, PDR remains stable. In these cases, the sample interval is long enough for every node to accomplish its transmission before the next sensor data is received. As the sample rate increases, the number of sensor data need to be 
sent per unit time augments and $P D R$ begins to decrease due to the increase of packet collisions. A single communications failure occurs when a device transaction fails to reach the coordinator, i.e., channel access failure after macMaxCSMABackoffs attempts or an acknowledgment is not received after macMaxFrameRetries attempts. $P D R$ with bigger BO begins to decrease first, because SO is the same and a bigger BO means that one sample interval includes less number of active portions.

Effect of beacon tracking. The average absolute deviation of $P D R$ between the tracking and notracking modes is less than $0.1 \%$ which is the simulation error. The two modes should have the same $P D R$, since the only difference between them is whether to track the beacon during the period after a transmission and before a new sample cycle and transmissions are not impacted.

5.1.2. Average Latency Fig. 3 presents the results of latency. The average absolute deviation of $A L$ between the tracking and notracking modes is $2.4 \%$.

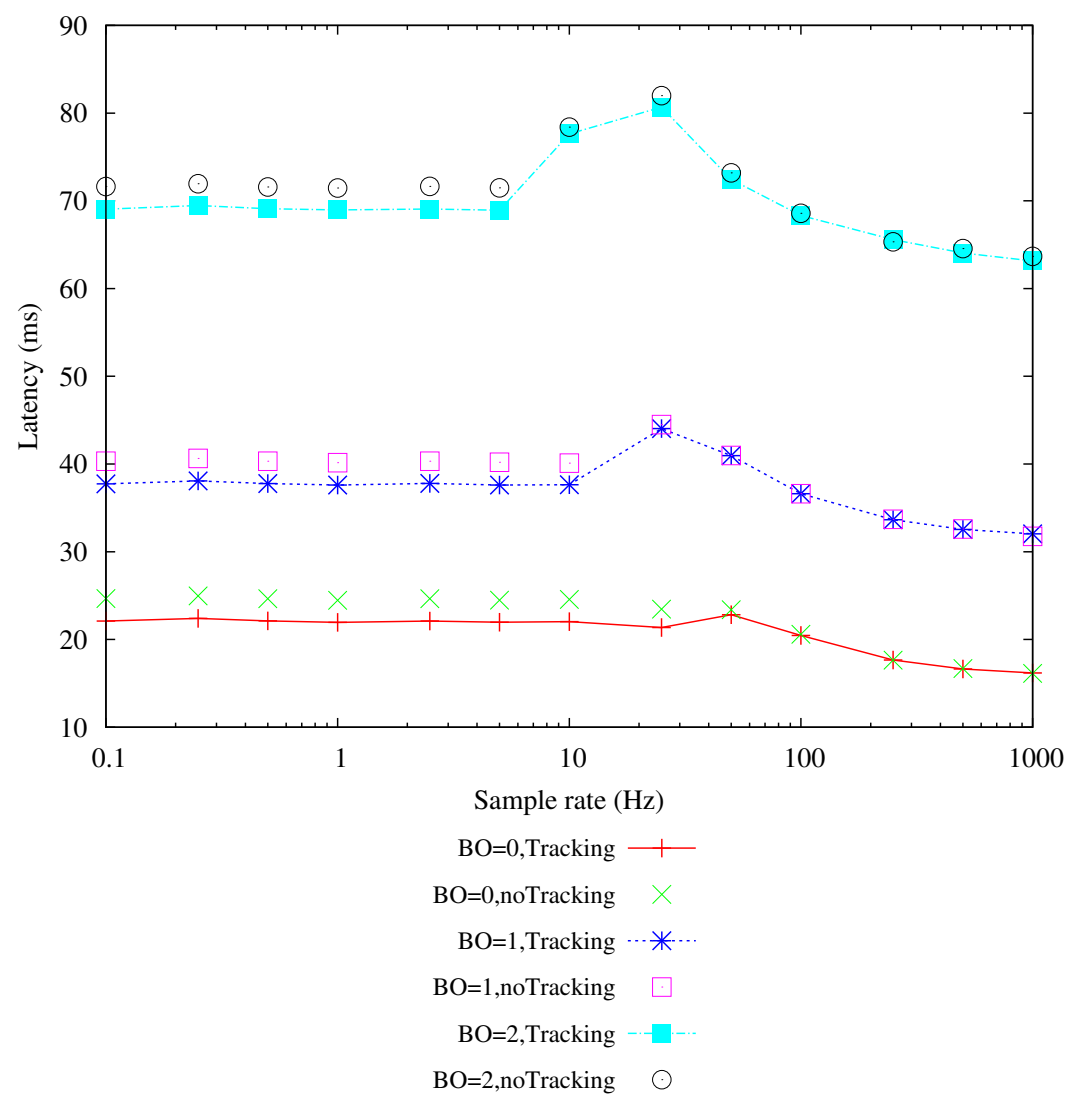

Figure 3. Latency of slotted CSMA-CA with fixed $S O$ and various $B O$

Effect of traffic load. The system of both tracking and notracking modes goes through 3 different stages, i.e., lightly loaded, heavily loaded and saturated. The tendency in change of latency of both modes are the same. During the lightly loaded stage, $A L$ remains stable because the transmissions between two adjacent sample intervals do not overlap; the network utilization is almost the same for different sample intervals.

When the system is heavily loaded, it is transiting to be saturated. $A L$ increases if B0 is not equal to $\mathrm{SO}$; on the contrary, it decreases slightly if $\mathrm{B} 0$ is 0 . In this transition period, some nodes 
can not complete their transmissions before the next sample interval begins and the last one or two nodes transmitting during the overlapping period of two sample intervals have to compete with other seven or six nodes for channel usage. If these last transmissions succeed, they will be longer than the case there were no overlapping between two sample intervals and $A L$ will increase compared with the lightly loaded stage; if they fail, they will not be considered in the calculation of latency and $A L$ will decrease. In addition, the new sensor data of the last one or two nodes will be delayed for transmitting, which extends $A L$. For the case that $\mathrm{BO}$ is 0 , there is no inactive portion in the superframe; thus the delay of new sensor data transmissions and the extension of old sensor data transmissions are smaller than the loss of failed transmissions of old sensor data, as a result, $A L$ decreases. However when $\mathrm{BO}$ is 1 and 2, each superframe comprises an inactive portion, especially in case that $\mathrm{BO}$ is 2 , the length of superframe is $61.44 \mathrm{~ms}$ which includes an inactive portion of $46.08 \mathrm{~ms}$; thus the delay of new sensor data transmissions and the extension of old sensor data transmissions are larger than the loss of failed transmissions of old sensor data.

As the sample rate continues to increase, the system becomes completely saturated. In these cases, all nodes always have pending sensor data to be sent and they compete for channel usage during the active portion of every superframe. If a node gets two new sensor data before the end of a transmission, only the last sensor data will be sent and first ones are discarded. The delay of new sensor data transmissions is small; therefore, $A L$ decreases.

The $A L$ of a bigger $\mathrm{BO}$ is larger than a smaller $\mathrm{BO}$, because $\mathrm{SO}$ is set to 0 . If some nodes can not transmit their sensor data in one active portion of a superframe, they have to wait at least one inactive portion to resume their transmissions. A bigger BO causes a longer inactive portion for waiting.

Effect of beacon tracking. The $A L s$ of notracking mode are a little bigger than those of tracking mode, which is caused by the timing cost of resynchronization. With notracking mode, nodes go to sleep mode after a transmission if they have no data to send. They wake up some time later and performs a new sensing operation. They need to be resynchronized with the coordinator before transmitting the new sensor data. The microcontroller wakes the transceiver up to acquire a beacon packet. During the transition from sleep to active mode of transceiver, the coordinator may be transmitting a beacon packet. The nodes will miss this beacon packet and they have to listen the channel for almost one superframe duration to receive the next beacon. This resynchronization process can be observed in the example of simulation log presented in Listing. 1.

Listing 1: Log fragment of noTracking mode

Node 0: wakes up to read sensor data at 8598154 us.

Node 0: reads a new sensor data (248) at 8600066 us

Node 0: wakes its transceiver up at 8600066 us .

Transceiver 8: transmits a beacon at 8601600 us

Proxy8: begins emitting packet at 8601792 us.

Proxy 8: completes emitting packet at 8602336 us .

Transceiver 0 : is woken up at 8602066 us

Node 0: transmits the data to the transceiver $\left({ }_{-} N R=0\right)$ with data $=248$ at 8602066 us

Transceiver 0: starts to track beacon at 8602066 us

Transceiver 0: receives a TX packet from microcontroller at 8602446 us.

Transceiver 8: transmits a beacon at 8663040 us

Proxy 8: begins emitting packet at 8663232 us .

Proxy8: completes emitting packet at 8663776 us. 
Transceiver 0: receives a beacon packet and starts to transmit the TX packet at 8663776 us

In the example shown in Listing. 1, Transceiver8 belongs to the coordinator and $B O$ is set to 2, corresponding to $61.44 \mathrm{~ms}$ superframe. With the notracking mode, the nodes have to wake up their transceivers after reading a new sensor data; however, during the wakeup transition of transceiver, the coordinator has transmitted a beacon packet at $860.16 \mathrm{~ms}$. The nodes have to listen to the channel and receive a beacon packet after one superframe duration at $866.3776 \mathrm{~ms}$. If the tracking mode is used, this resynchronization will not occur. The transceiver uses a built-in timer to count for the superframe length and wake up automatically before the transmission of beacon packet. Listing. 2 illustrates the simulation log of the same scenario in the tracking mode.

\section{Listing 2: Log fragment of tracking mode}

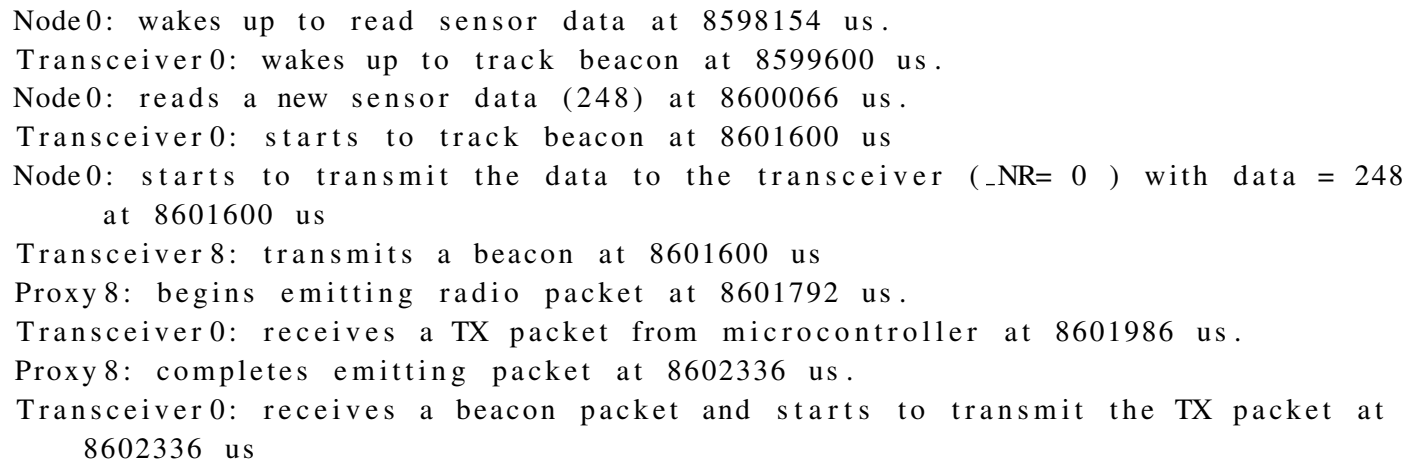

The beacon packet at $860.16 \mathrm{~ms}$ was received by the synchronized transceivers which are woken up for tracking beacon automatically by a built-in timer. Therefore, the latency of notraking mode is larger than tracking mode.

Note that the timing cost of resynchronization of the noTracking mode, compared to the tracking mode, only happens when a transmission of beacon packet occurs during the wakeup transition of transceiver. If there is no beacon packet transmission during the wakeup transition of transceiver, the latency results of notracking and tracking modes are the same; however, the power consumption of these two modes is still different. After reading a sensor data, the nodes of tracking mode can go to sleep again and wake up before the transmission of beacon packet, because they know the information of superframe. On the contrary, the nodes of notracking mode have to listen the channel until the receipt of a beacon packet.

\subsubsection{Average Power Consumption Fig. 4 demonstrates the simulation results of average power consumption.}

Effect of traffic load. During the lightly loaded stage, the APCs of notracking mode increase as the sample rate augments. In these cases, the nodes go to sleep mode after the transmission and wake up when the next sample interval begins, so the energy spent during one sample interval for different sample rate are almost the same. The difference of energy consumption between two sample rates is the length of sleep mode. Therefore, the $A P C$ s increases as the sample interval decreases. In addition, the $A P C$ of a bigger $B O$ is higher than that of a smaller $B O$, because the energy cost of resynchronization of notracking mode for a bigger $B O$ is more heavy. In these cases, after reading a new sensor data, the microcontroller turns on the transceiver to listen for a beacon. The listening 


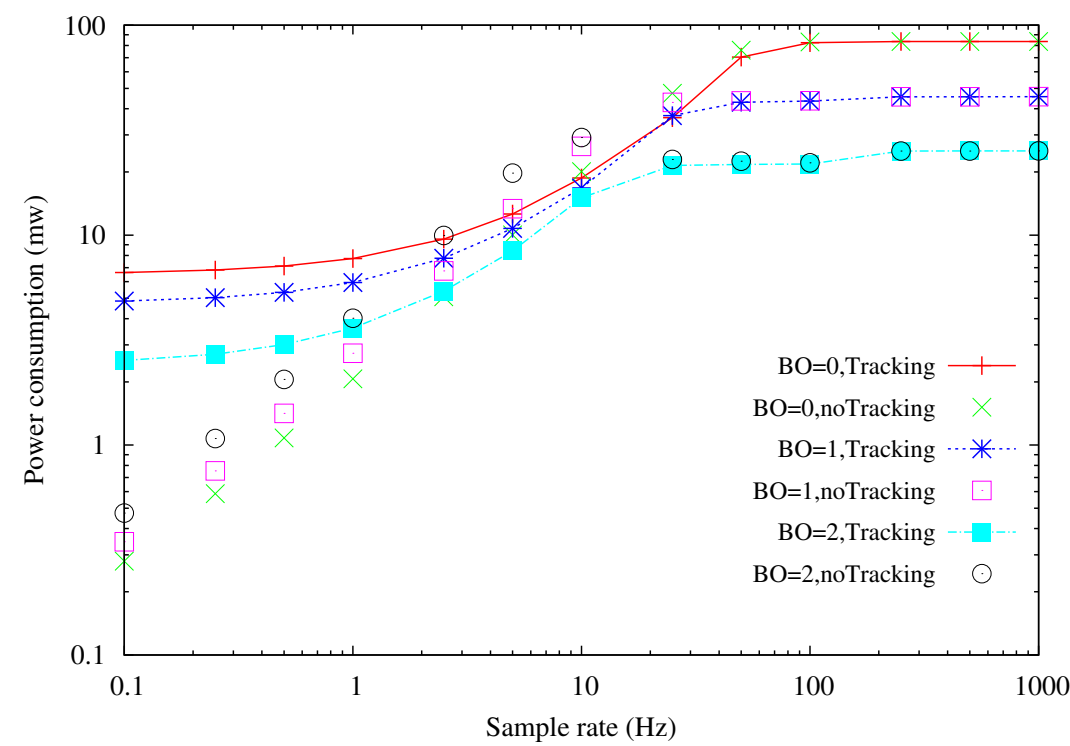

Figure 4. Power consumption of slotted CSMA-CA with fixed $S O$ and various $B O$

period is the cost of resynchronization which can be observed in Listing. 1. The transceiver of Node0 is woken up at $8602066 \mu$ s which is $60974 \mu$ s before the beacon packet transmission. The transceiver has to listen to the channel during this time. The listening period may last long if the length of superframe is big. Finally, $A P C$ is tending towards stability when the system is saturated. The microcontroller keeps in active mode and the transceiver goes to sleep mode during the inactive portion of a superframe and turn to active when active portion begins.

As the sample rate increases, $A P C$ s augments, because more sensor data need to be sent. For a same sample rate, the power consumption of a smaller $\mathrm{BO}$ is larger than a bigger $\mathrm{BO}$, since a smaller BO means more beacons received and shorter inactive portion of a superframe.

During the lightly loaded stage, $A P C$ s augments slowly. In these cases, most of the time, the nodes are in sleep mode. When the system is heavily loaded transiting to be saturated, sharp increase of $A P C$ s can be observed. During this period, the power consumptions of transmissions account for the main part of the whole power consumption. When the system becomes completely saturated, APCs become steady which are the largest power consumptions per node. The nodes are always in active mode during the active portion of a superframe. Because there is no inactive portion when BO is 0 , its power consumptions are bigger than others.

Effect of beacon tracking. The APCs of notracking mode are smaller than tracking mode during the lightly loaded stage, because one sample interval consists of too many superframes and the tracking mode spends too much energy for useless beacon tracking. When the system is heavily loaded, the $A P C$ s of notracking mode are much bigger than tracking mode. During this period, the cost of resynchronization of notracking mode is much bigger than the useless beacon tracking of tracking mode since one sample interval consists of a few of superframes. When the system becomes completely saturated, the two modes have the same behaviors and the APCs become steady.

5.1.4. Energy Consumption per Packet Fig. 5 illustrates the simulation results of energy consumption per packet. 


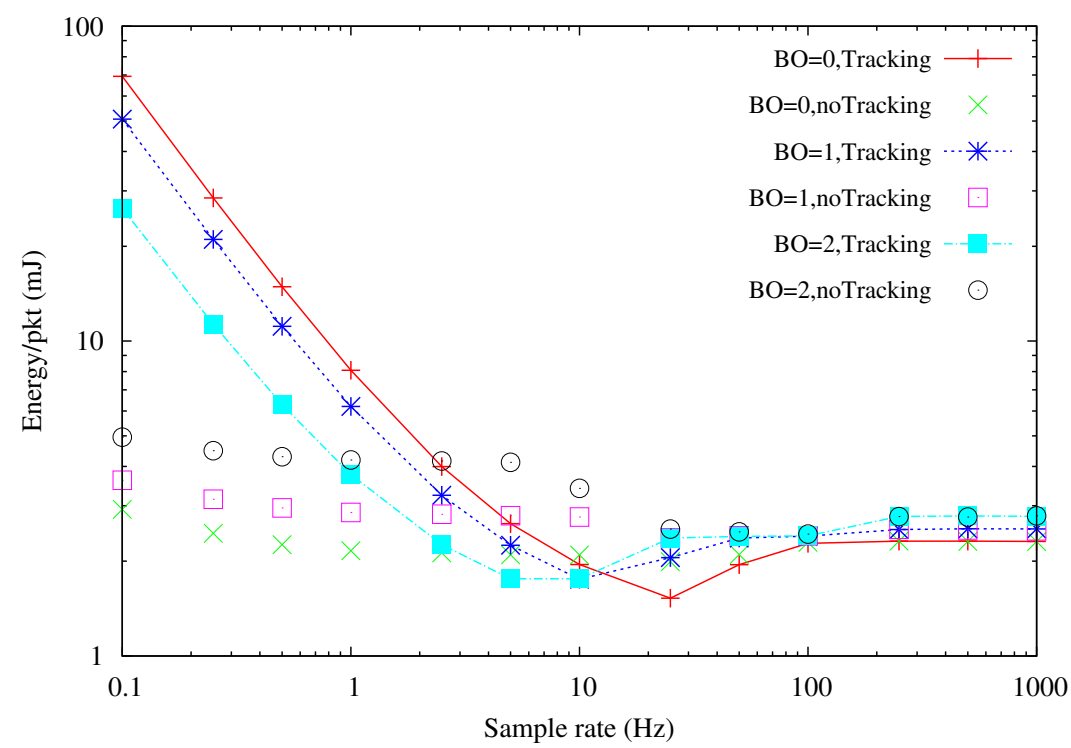

Figure 5. Energy consumption per packet of slotted CSMA-CA with fixed $S O$ and various $B O$

Effect of traffic load. During the lightly loaded stage, ECPkt decreases as the sample rate increases and the smallest BO consumes the most energy for a fixed sample rate. In these cases, the average number of packets transmitted during one sample interval is the same for different sample rates, which can be proved by the constant PDRs in Fig. 2; ECPkt is therefore less if the sample interval is shorter. For a fixed sample rate, a smaller BO consumes more energy since one sample interval includes more superframes and the nodes have to wake up to track the beacon packet at the beginning of each superframe.

The smallest ECPkt occurs when PDR begins to decline, where the system begins to transit to be saturated. In this case, every node can accomplish its transmission before new sensor data arrives, but the interval between the last node turns to sleep and the next sensor data arrives is very short, so the nodes spend the least energy in sleep mode. As the sample rate continues to increase, the ECPkt augments due to the transmission overlap of two sample intervals and the increase of collisions. The energy consumption per packet with bigger BO begins to increase first because the number of superframes per sample interval is less for a larger BO.

When the system is saturated, ECPkt remains constant and the smallest BO consumes the least energy. In these cases, nodes are always having pending sensor data to send. The number of successfully transmitted packets per superframe is almost the same; therefore ECPkt remains constant for the same BO. In addition, for a fixed sample rate, because one superframe includes a longer inactive portion if $\mathrm{BO}$ is larger, its $E C P k t$ is higher.

Effect of beacon tracking. During the lightly loaded stage, the ECPkts of notracking mode remain constant, because the energy spent during one sample interval for different sample rate are almost the same and the numbers of successfully transmitted packet during one sample interval for different sample rates are same. While the system is heavily loaded, ECPkt begins to decrease because the cost of resynchronization declines. Finally, ECPkt is tending to stability when the system is saturated. 
When the sample rate is small $(0.1,0.25,0.5,1$ and 2.5 for $B O$ set to 1$)$, the ECPkts of notracking mode are smaller than the tracking mode, because the useless beacon tracking consumes much energy. At the end of lightly loaded stage and the entire transition stage, the ECPkts of notracking mode are higher than the tracking mode, since the resynchronization of notracking mode consumes much energy. When the system is saturated, the ECPkts of notracking and tracking mode are the same. In these cases, the system behaviors of the two modes are the same.

5.1.5. Overall Analysis Based on the above analysis, we can find a best parameter setting for different sample rates. If the sample rate is small, the notracking mode with $B O$ set to 0 consumes the least energy for transmitting one packet and provides the shortest $A L$ with the best $P D R$. When the sample rate is 25 , the tracking mode with $B O$ set to 0 performs better than any other settings. If the sample rate is larger than 25 , for this application with 8 nodes and 1 coordinator, the performance of these two modes are the same. In this period, the contention-based CSMA-CA algorithm cannot guarantee a $P D R$ higher than $95 \%$ and $B O$ should be set to 0 in order to provide biggest $P D R$ with shortest $A L$ and smallest $E C P k t$; ; however, these advantages are obtained by sustaining a big power consumption which results a short lifetime of sensor nodes and the network.

The beacon-enabled mode without beacon tracking and with $S O$ set to 0 has successfully solved the useless beacon tracking problem. The ECPkts and APCs become smaller when the sample rate is smaller. However, it still has a problem that the ALs of a larger $B O$ are much longer than a smaller $B O$, which is caused by the waiting in the inactive portion of a superframe because $S O$ is set too small and some transmissions can not be finished in one superframe. Therefore, in next section, the same simulations will be re-executed with $S O$ set to the same value with $B O$.

\subsection{Slotted CSMA-CA with Equal SO and BO}

In the last section, $S O$ is fixed to 0 and variable $B O$ results different duty cycle in a superframe. In this section, the application is implemented by using the beacon-enabled slotted CSMA-CA algorithm without beacon tracking and with a $S O$ that is equal to $B O$; the inactive portion of the superframe is thus 0 . The unfinished transmissions do not have to wait a long inactive portion for resuming. The simulation results are presented in Fig. 6 to Fig. 9.

In order to facilitate the comparisons, the simulation results of beacon-enabled mode without beacon tracking and with $S O$ set 0 are also illustrated in Fig. 6 to Fig. 9. Two improvements have been obtained if $S O$ is set to the same value with $B O$. They are presented as follows.

- PDR: $P D R$ s with $B O$ set to 1 and 2 are increased when the sample rate is larger than the start point of the stage of transition to be saturated. Because $S O$ is equal to $B O$, the nodes have more time to transmit the sensor data and the start point of the system heavily loaded is deferred.

- $A L$ : Latency diminishes, since the inactive portion of a superframe is set to 0 and the nodes do not have to wait a long time for resume their transmissions in a new superframe. During the lightly loaded stage, the ALs of a larger $B O$ are larger than a smaller $B O$. In these cases, the nodes are in sleep mode if they have no sensor data to be sent. They will be woken up by a built-in timer if they need to read a sensor data. In the beacon-enabled mode, the nodes can only transmit the new sensor data after they successfully receive a beacon packet. We name 


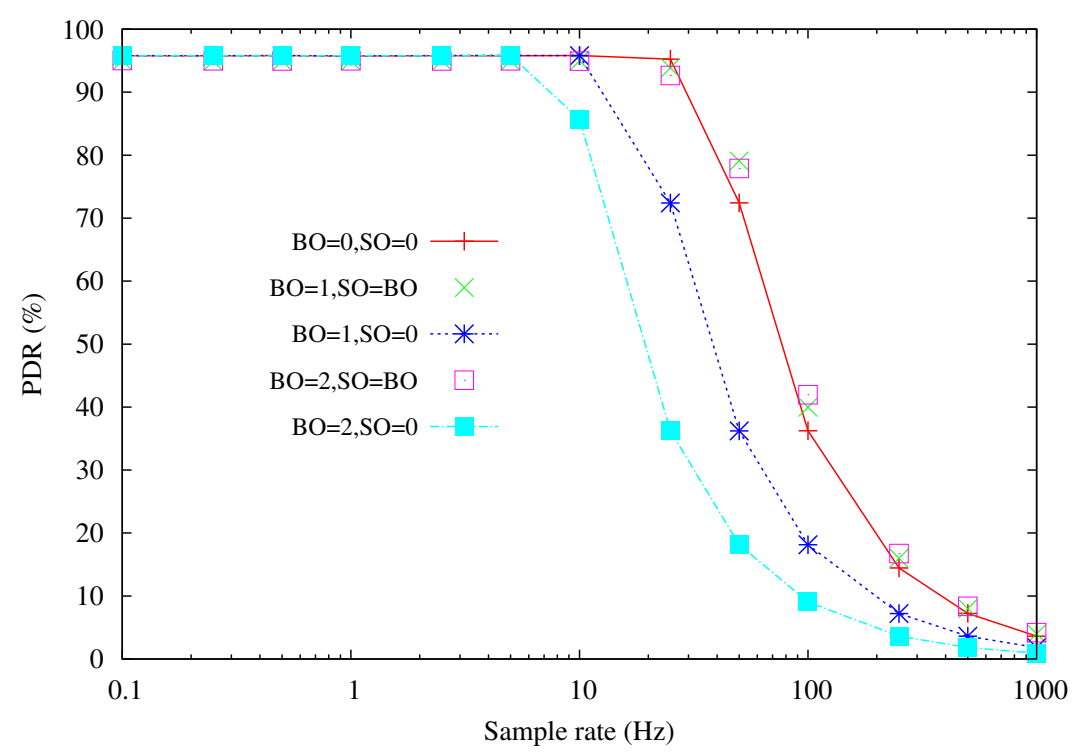

Figure 6. Packet delivery rate of slotted CSMA-CA with SO equal to BO

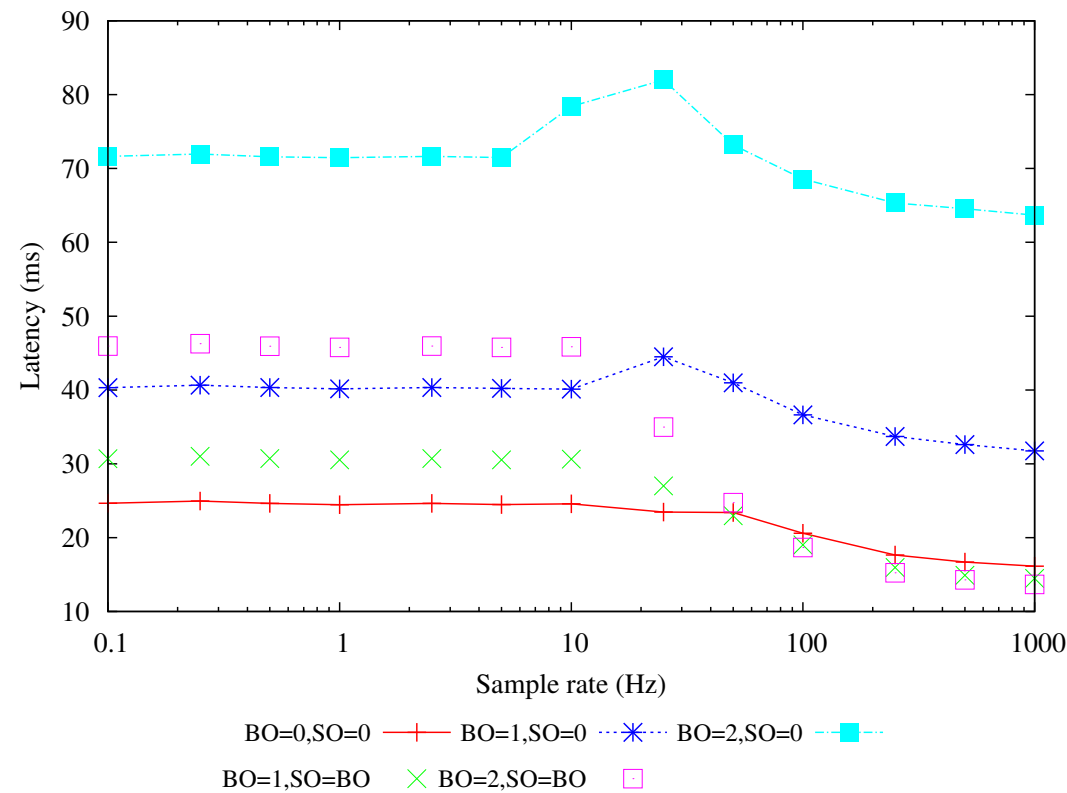

Figure 7. Latency of slotted CSMA-CA with SO equal to BO

this kind of delay of latency is the cost of resynchronization. A sensor data may wait for a longer time to be sent if $B O$ is bigger. When the system is heavily loaded, the ALs become small. As the system becomes completely saturated, the ALs of a larger $B O$ are shorter than a smaller $B O$ as a result of less receiving of beacon. 


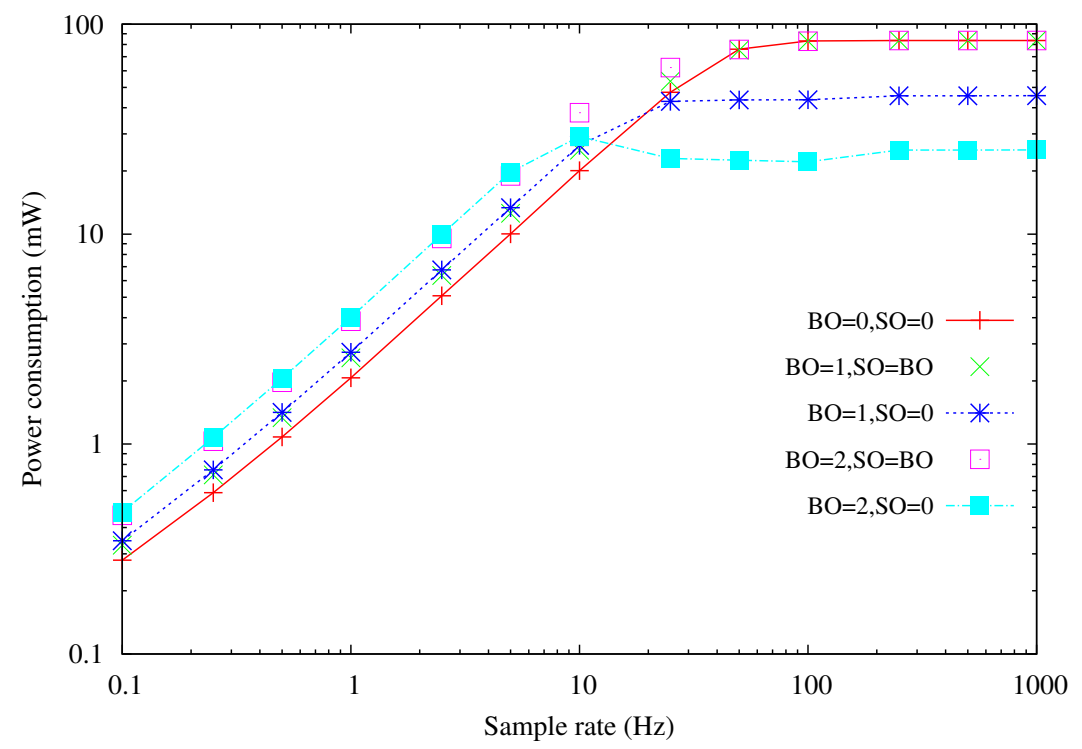

Figure 8. Power consumption of slotted CSMA-CA with SO equal to BO

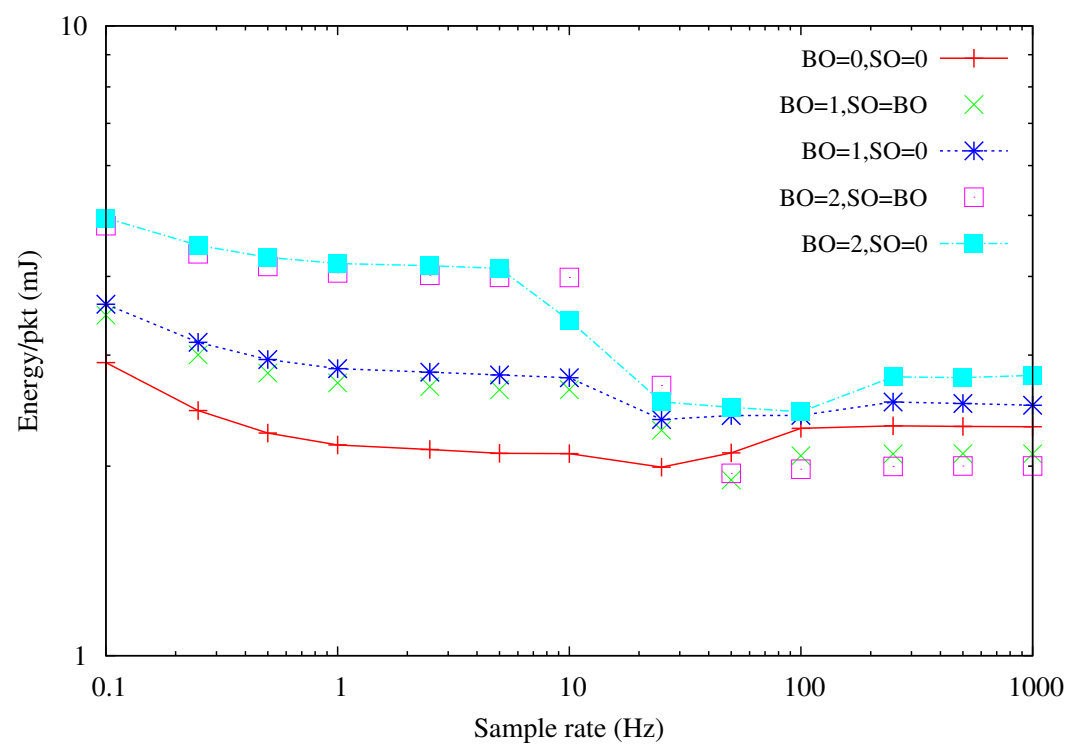

Figure 9. Energy consumption per packet of slotted CSMA-CA with SO equal to BO

\subsection{Unslotted CSMA-CA}

The cost of resynchronization is an intrinsic drawback of beacon-enable mode; in this section, the performance of nonbeacon-enabled mode with unslotted CSMA-CA algorithm is thus evaluated. The simulation results are shown in Fig. 10 to Fig. 13.

The simulation results of beacon-enabled mode without beacon tracking and with $S O$ set 0 are also illustrated in Fig. 10 to Fig. 13. Without the cost of resynchronization, the nonbeacon-enabled mode provides smaller ALs than beacon-enabled mode; in addition, the ECPkts and APCs of nonbeaconenabled mode are smaller if the system is not saturated. However, the PDRs of nonbeacon-enabled mode are smaller than beacon-enabled mode. Because the transmissions of nonbeacon-enabled 


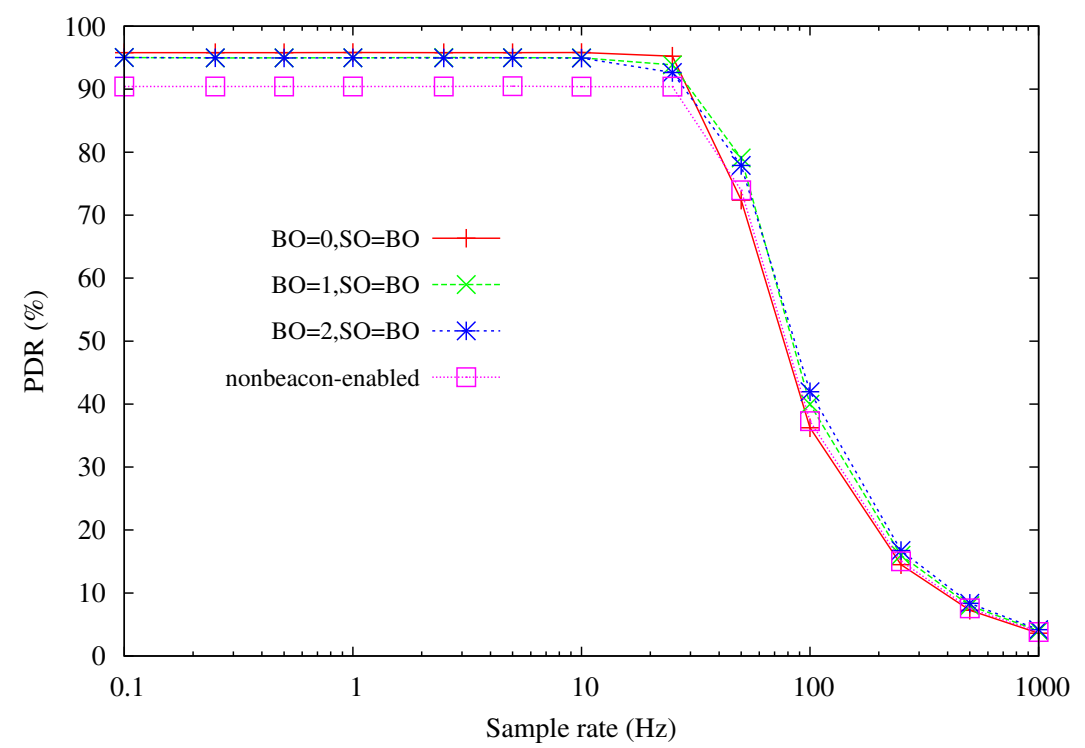

Figure 10. Packet delivery rate of unslotted CSMA-CA in nonbeacon-enabed mode

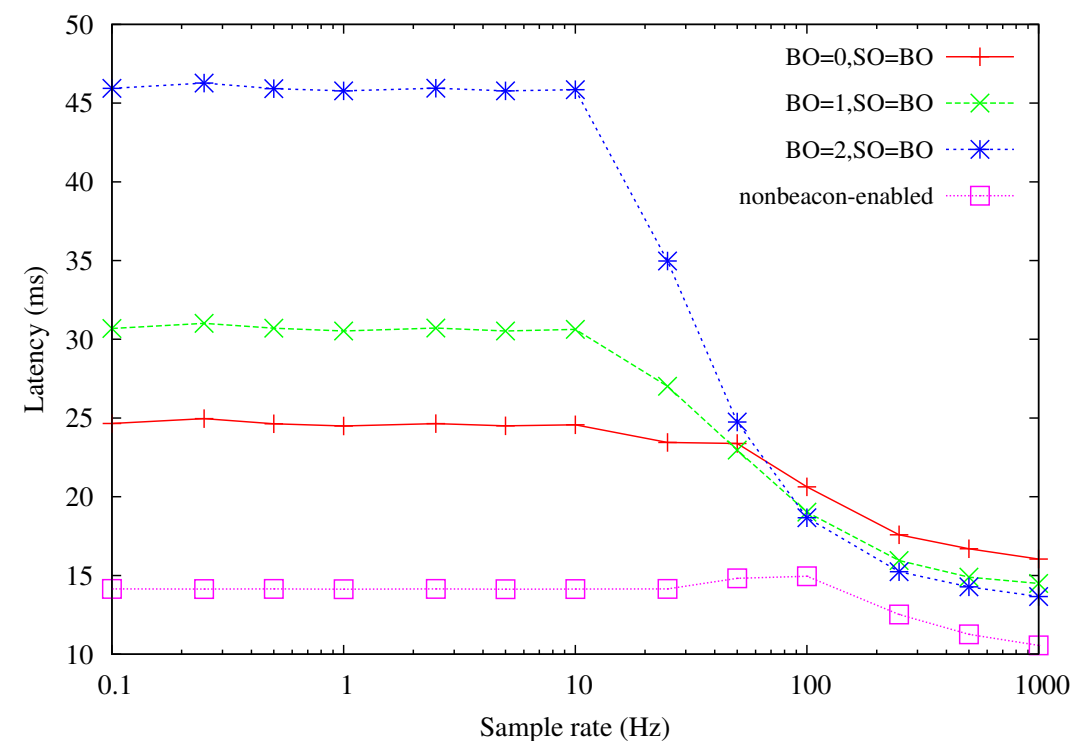

Figure 11. Latency of unslotted CSMA-CA in nonbeacon-enabed mode

mode are not aligned with the backoff period boundary and CW is set to 0 , the number of collisions is increased. When the system is saturated, the ECPkts of nonbeacon-enabled mode are higher due to its smaller $P D R$ s.

\subsection{Summary of all Simulation Results}

Based on the above simulations and analysis, we find the following conclusions which are useful to the configurations of IEEE 802.15.4 sensor networks in industrial applications.

- The nonbeacon-enabled mode can provide better performance in the aspects of latency and energy consumptions than beacon-enabled mode, but with a smaller PDR. 


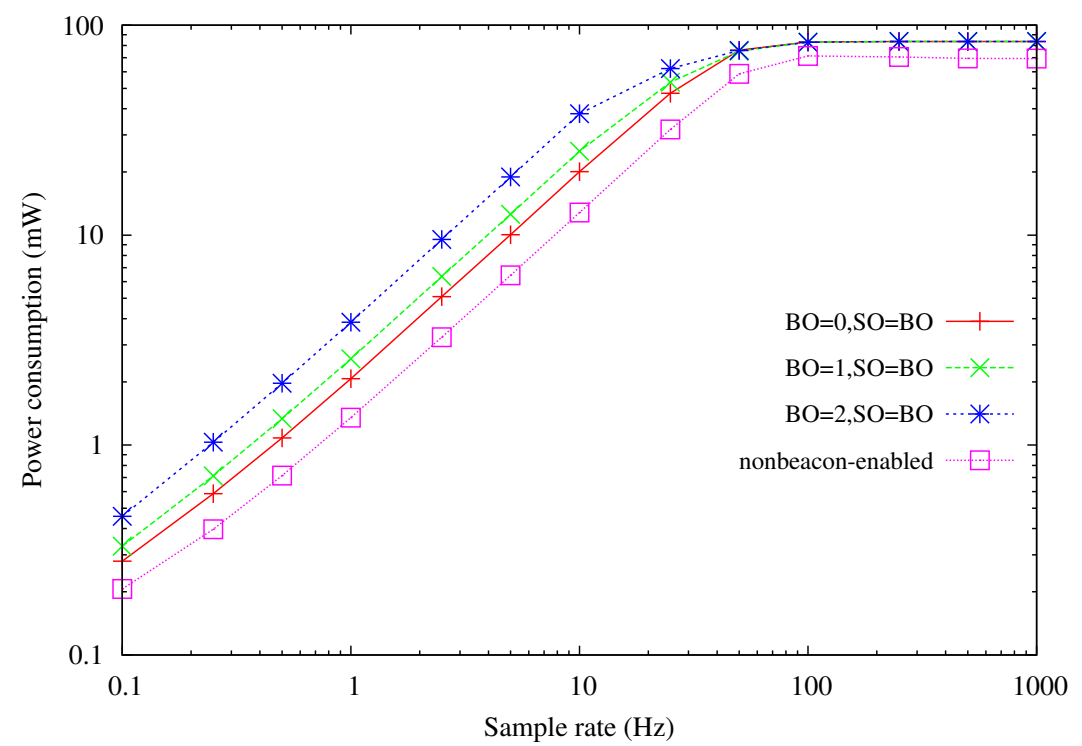

Figure 12. Power consumption of unslotted CSMA-CA in nonbeacon-enabed mode

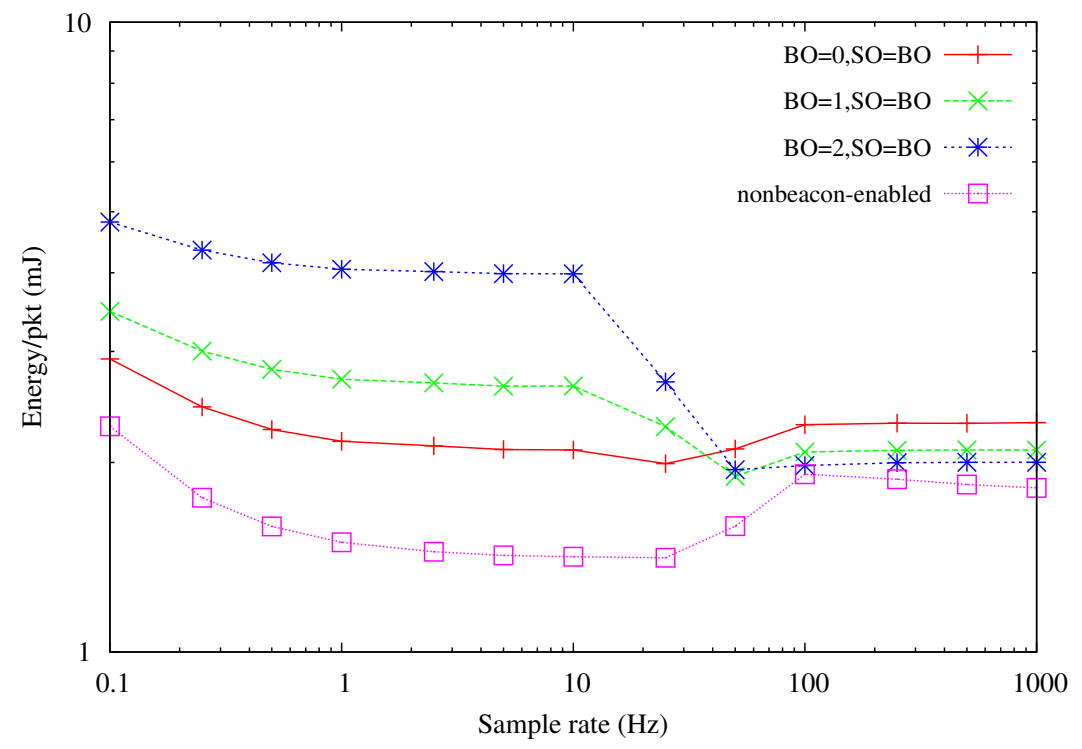

Figure 13. Energy consumption per packet of unslotted CSMA-CA in nonbeacon-enabed mode

- The beacon-enabled mode offers a higher PDR; it has nevertheless one intrinsic cost of resynchronization which increases the latency and power consumption.

- If the sample rate is small and the system is lightly loaded, the nodes spend too much for many useless beacon receipts if tracking is specified; thus notracking mode should be used. In order to minimize the impact of the resynchronization cost, $B O$ is set to 0 .

- During the period that the system is heavily loaded, if $S O$ is set to the values of $B O$, the start point of the transition can be deferred. In addition, the beacon-enabled mode with beacon tracking can support a better ECPkt. 
- When the system is completely saturated, the PDRs of the CSMA-CA algorithms are less than $50 \%$ and a larger $B O$ with $S O$ set to the same value can provide a better performance in the aspects of both latency and energy consumption.

\section{CONCLUSION}

In this paper, we evaluate the performance of IEEE 802.15.4 sensor network in industrial applications. To provide high accurate modeling of software execution and wireless sensor hardware platform, IDEA1 is used as the simulator which is based on the hardware and software co-simulation of SystemC. The simulation results of IDEA1 are convincing since it considers many details of hardware and software executions in simulation and is validated by testbed experiments. The widely-used hardware platform, MICAz, is modeled by SystemC in IDEA1. IEEE 802.15.4 PHY and MAC protocols are implemented in IDEA1 with relative energy consumption parameters. The main functions of the MAC layer in this standard are studied based on the PHY settings. Both the slotted CSMA-CA protocol in beacon-enabled mode and the unslotted CSMA-CA protocol in nonbeacon-enabled mode are analyzed in the aspects of packet delivery rate, latency, power consumption per node and energy consumption per packet. Two synchronization mechanisms in the IEEE 802.15.4 standard, the beacon tracking and non beacon tracking, are implemented. The effect of various parameter settings, especially the beacon order and the superframe order, are analyzed under different traffic loads. Different combinations of these settings offer various performance in aspects of packet delivery and energy consumption. For instance, the beacon-enabled mode offers a higher $P D R$; the nonbeacon-enabled mode can provide better latency and energy consumptions since it does not need resynchronization. Through the extensive simulations in this work, proper settings of transmission mode and parameters are found for different application requirements and traffic loads. Although the object of this work is to evaluate the feasibility of using IEEE 802.15.4 protocols in our project about the measurement and control of automobile vibrations and noise, since no specific parameter of this application is used in the study, the simulation results of various configurations and the analysis conclusion can be used in any networks with the same star topology which is a common network structure in industrial applications.

\section{REFERENCES}

1. Mieyeville F, Ichchou M, Scorletti G, Navarro D, Du W. Wireless sensor networks for active vibration control in automobile structures. Smart Materials and Structures 2012; 21(7):075 009.

2. Chen F, Talanis T, German R, Dressler F. Real-time enabled IEEE 802.15.4 sensor networks in industrial automation. 2009 IEEE Int. Symposium on Industrial Embedded Systems, IEEE, 2009; 136-139, doi:10.1109/SIES. 2009.5196207.

3. Institute of Electrical and Electronics Engineers. IEEE Standard for Information technology- Telecommunications and information exchange between systems- Local and metropolitan area networks- Specific requirements Part 15.4: Wireless Medium Access Control (MAC) and Physical Layer (PHY) Specifications for Low-Rate Wireless Personal Area Networks (WPANs) 2006.

4. Crossbow Technology, Inc.. MICAz Datasheet. URL www.xbow.com/Products/Product_pdf_files/ Wireless_pdf/MICAz_Datasheet.pdf. 
5. Fall K, Varadhan K. The ns Manual (formerly ns Notes and Documentation) Jan 2009. URL http: //www . isi . edu/nsnam/ns/doc/ns_doc.pdf.

6. Varga A. The omnet++ discrete event simulation system. Proc. of the European Simulation Multiconference (ESM'2001) June 2001; .

7. Kostin A, Oz G, Haci H. Performance study of a wireless mobile ad hoc network with orientation-dependent internode communication scheme. International Journal of Communication Systems 2012;

8. Levis P, Lee N, Welsh M, Culler D. Tossim: accurate and scalable simulation of entire tinyos applications. Proc. of the 1st int. conf. on Embedded networked sensor systems, SenSys '03, ACM: New York, NY, USA, 2003; 126-137.

9. Titzer BL, Lee DK, Palsberg J. Avrora: scalable sensor network simulation with precise timing. Proc. of the 4th international symposium on Information processing in sensor networks, IPSN '05, IEEE Press: Piscataway, NJ, USA, 2005.

10. Du W, Mieyeville F, Navarro D, Connor IO. IDEA1: A validated systemc-based system-level design and simulation environment for wireless sensor networks. EURASIP Journal on Wireless Communications and Networking 2011; $: 1-20$.

11. Institute of Electrical and Electronics Engineers. IEEE Std 1666 - 2005 IEEE Standard SystemC Language Reference Manual 2006.

12. Zheng J, Lee MJ. Will IEEE 802.15.4 make ubiquitous networking a reality?: a discussion on a potential low power, low bit rate standard. Communications Magazine, IEEE Jun 2004; 42(6):140-146, doi:10.1109/MCOM. 2004.1304251.

13. Zheng J, Lee MJ. A comprehensive performance study of ieee 802.15. 4. Sensor network operations 2004; :218237.

14. Ramachandran I, Das AK, Roy S. Analysis of the contention access period of ieee 802.15.4 mac. ACM Trans. Sen. Netw. March 2007; 3, doi:http://doi.acm.org/10.1145/1210669.1210673.

15. Lu G, Krishnamachari B, Raghavendra CS. Performance evaluation of the ieee 802.15. 4 mac for low-rate lowpower wireless networks. Performance, Computing, and Communications, 2004 IEEE International Conference on, IEEE, 2004; 701-706.

16. Petrova M, Riihijarvi J, Mahonen P, Labella S. Performance study of ieee 802.15. 4 using measurements and simulations. Wireless Communications and Networking Conference, 2006. WCNC 2006. IEEE, vol. 1, IEEE, 2006; 487-492.

17. Rohm D, Goyal M, Hosseini H, Divjak A, Bashir Y. A simulation based analysis of the impact of ieee 802.15. 4 mac parameters on the performance under different traffic loads. Mobile Information Systems 2009; 5(1):81-99.

18. Chen F, Wang N, German R, Dressler F. Simulation study of ieee 802.15.4 1r-wpan for industrial applications. Wireless Communications and Mobile Computing 2010; 10(5):609-621, doi:10.1002/wcm.736.

19. Xia F, Vinel A, Gao R, Wang L, Qiu T. Evaluating ieee 802.15 .4 for cyber-physical systems. EURASIP J. Wirel. Commun. Netw. 2011; 2011, doi:http://dx.doi.org/10.1155/2011/596397.

20. Nordin N, Dressler F. On the impact of beacon collisions in co-located ieee 802.15. 4-based networks. IEEE INFOCOM, 2012.

21. Ullah S, Higgins H, Shen B, Kwak KS. On the implant communication and mac protocols for wban. International Journal of Communication Systems 2010; :982-999doi:10.1002/dac.v23:8.

22. Liang X, Balasingham I. Performance analysis of the ieee 802.15. 4 based ecg monitoring network. Proceedings of the 7th IASTED International Conferences on Wireless and Optical Communications, 2007; 99-104.

23. Pollin S, Ergen M, Ergen S, Bougard B, Der Perre L, Moerman I, Bahai A, Varaiya P, Catthoor F. Performance analysis of slotted carrier sense ieee 802.15. 4 medium access layer. Wireless Communications, IEEE Transactions on 2008; 7(9):3359-3371.

24. Buratti C. Performance analysis of ieee 802.15. 4 beacon-enabled mode. Vehicular Technology, IEEE Transactions on 2010; 59(4):2031-2045.

25. Du W, Navarro D, Mieyeville F. A simulation study of ieee 802.15. 4 sensor networks in industrial applications by system-level modeling. Proc. of the Fourth International Conference on Sensor Technologies and Applications (SENSORCOMM), 2010; 311-316.

26. Du W, Mieyeville F, Navarro D. Modeling energy consumption of wireless sensor networks by systemc. Proc. of the Fifth International Conference on Systems and Networks Communications (ICSNC), 2010; 94-98.

27. Ullah N, Sanaullah Chowdhury M, Khan P, Ullah S, Kwak KS. Throughput and delay limits of chirp spread spectrum-based ieee 802.15. 4a. International Journal of Communication Systems 2012; 25(1):1-15.

28. Sanaullah Chowdhury M, Ullah N, Ameen MA, Kwak KS. Framed slotted aloha based mac protocol for low energy critical infrastructure monitoring networks. International Journal of Communication Systems 2012; .

29. Lee YD, Jeong DU, Lee HJ. Empirical analysis of the reliability of low-rate wireless u-healthcare monitoring applications. International Journal of Communication Systems 2013; 26:505-514. 
30. Bhunia S, Misra IS, Sanyal SK, Kundu A. Performance study of mobile wimax network with changing scenarios under different modulation and coding. International Journal of Communication Systems 2011; 24(8):1087-1104.

31. Liu W, Jin H, Wang X, Guizani M. A novel ieee 802.11-based mac protocol supporting cooperative communications. International Journal of Communication Systems 2011; 24(11):1480-1495.

32. Kułakowski P, Calle E, Marzo JL. Performance study of wireless sensor and actuator networks in forest fire scenarios. International Journal of Communication Systems 2013; 26:515-529.

33. ZigBee Alliance. ZigBee specification - Document 053474r17 2007. URL http: / /www . zigbee. org/.

34. Koubaa A, Alves M, Tovar E. Energy and delay trade-off of the gts allocation mechanism in ieee 802.15. 4 for wireless sensor networks. International Journal of Communication Systems 2007; 20(7):791-808.

35. Du W, Navarro D, Mieyeville F, O'connor I. IDEA1: A validated systemc-based simulator for wireless sensor networks. Proc. of IEEE 8th International Conference on Mobile Adhoc and Sensor Systems (MASS), 2011; 825830.

36. Savvides A, Park S, Srivastava MB. On modeling networks of wireless microsensors. Proceedings of the 2001 ACM SIGMETRICS international conference on Measurement and modeling of computer systems, SIGMETRICS '01, ACM: New York, NY, USA, 2001; 318-319, doi:http://doi.acm.org/10.1145/378420.378808. URL http: //doi.acm.org/10.1145/378420.378808.

37. Samimi H. Performance analysis of lognormally shadowed generalized gamma fading channels. International Journal of Communication Systems 2011; 24(1):14-26.

38. Black DC, Donovan J. SystemC: From the Ground Up. Springer-Verlag New York, Inc.: Secaucus, NJ, USA, 2005.

39. Atmel Corporation. ATMEL ATMega128 datasheet. URL www atmel.com/atmel/acrobat/doc2467. pdf.

40. Texas Instruments Incorporated. TI CC2420 datasheet. URL focus.ti.com/lit/ds/symlink/cc2420. pdf. 